\title{
Lossy Compression of Color Image Using EZW and BTC
}

\author{
Ajay Singh Yadav ${ }^{1}$, Halima Sadia ${ }^{2}$, Shashank Singh ${ }^{3}$ \\ ${ }^{1,2,3}$ Department of CSE, Integral University, Lucknow, India
}

\begin{abstract}
The images and videos are not only a medium of entertainment but also use for study and informational purpose. But these multimedia files consume huge memory space in hard disk as well as over the network, in order to save memory space we need to compress it. In beginning compression algorithms remove redundant data but now we have techniques like DWT (Discrete Wavelet Transform). Image compression algorithms like EZW, LZW, SPIHT are base on wavelets transform but initially they work on grayscale images. Hybrid image compression algorithms, where we hybridize two or more algorithm for better result, have been use for many times to provide efficient compression. In this paper, a hybrid algorithm is proposed which use EZW and BTC algorithm to compress a color image with the help of Huffman coding, here Huffman coding is use for the entropy coding. This hybrid system uses the wavelet decomposition to compress a color image and try to improve the results.
\end{abstract}

Keywords: DWT (Discrete Wavelet Transform), EZW(Embedded Zero Tree Wavelet Transform), BTC(Block Truncation Coding), Huffman Coding

\section{Introduction}

Now a days everything is going to digital and so are the mediums of presenting information. Multimedia files are become the new and strong medium to present the piece of information, which is not only attractive as well as easy to understand and to explain. The image and video files have become the most common and widely use media for not only entertainment as well as for corporate world, study and research.

But the problems with these files are they occupy a large amount of memory space in hard disk and over the network. To save space and make efficient use of these file we have to compress it. This size of data is because it has redundancies present in it. The reduction of that unwanted data is called compression. When we compress the data is called compression and to gat original file back from this compressed data is called decompression.

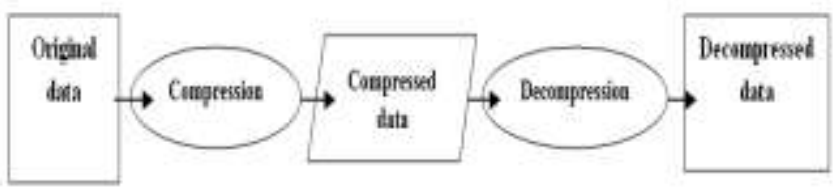

Figure 1: Block Diagram to show basic functionality of Compression/Decompression Algorithm

In beginning compression algorithms remove redundant data but now we have techniques like DWT (Discrete Wavelet Transform). Data compression is of two types mainly, one is Lossless data compression and second one is Lossy data compression.

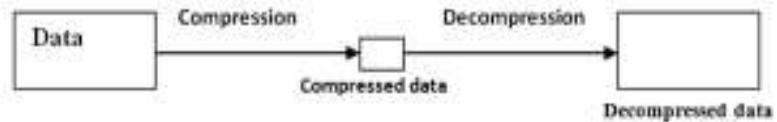

(a) Lossy Data Compression

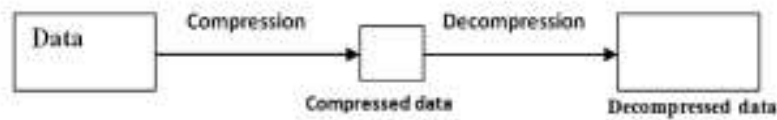

(b) Lossless Data Compression

Figure 2: Small size of compressed data in lossy (a) Vs large size of compressed data in lossless (b)

\section{A. Lossy Data Compression}

In lossy data compression when the decompression is performed there always some loss of the data which means we are unable to get the $100 \%$ original data from the compressed file. This loss varies from algorithm to algorithm as well as data to data.

\section{B. Lossless Data Compression}

In Lossless data compression when the decompression is done there is no loss of data; we can say that we are able to get the original data back from the compressed data.

The lossy image compression offers more Compression ratio than lossless image compression. It means it compressed data after compression with lossy image compression, takes less memory space or bandwidth in comparison to lossless data compression. In many cases the lossy image compression algorithm is faster than lossless.

The image compressed by lossless image compression takes more space but after decompression is almost as same as the original image.

In this paper we use EZW and BTC to compress a color image. A color image is made up of RGB planes in order to easily compress the RGB we convert it into $\mathrm{YCbCr}$ planes. And apply EZW and BTC algorithm separately in all planes to get the compression done. Huffman coding is use as

Volume 6 Issue 12, December 2017 


\section{International Journal of Science and Research (IJSR) \\ ISSN (Online): 2319-7064}

Index Copernicus Value (2016): 79.57 | Impact Factor (2015): 6.391

entropy coding in this proposed hybrid algorithm. The performance of algorithm is measured by Peak Signal to Noise Ratio and CR.

\section{EZW}

Embedded Zero trees of wavelet transform (EZW), developed by J. Shapiro in 1993, is similar to SPIHT algorithm but simpler. EZW is a lossy algorithm which provide high rate of compression. EZW algorithm use wavelet decomposition to provide subbands coefficient which mostly in form of one, zero or nearly zero. The transformed coefficients are considered as a tree (or trees) with the coefficients which have smallest frequency are put at the root node of the tree and the children of each node is a related coefficient in the next subband of higher frequency in comparison to the parent note, some trees or subtrees often have elements which is zero or close to zero, these subtrees are called zerotrees. Here the bits are assigned according to the importance of the nodes, generating a fully embedded code. This embedded code represent a sequence of binary $(0,1)$ numbers that differentiate the image data from 'null' image data.

\section{BTC}

Block Truncation Coding (BTC) is a lossy image compression technique. BTC is initially design only for grayscale images it means its traditional algorithm cannot work on color images here we use BTC and compressed a color image because we convert out color image into $\mathrm{YCbCr}$ plane and apply BTC on these planes separately. BTC breaks image into blocks of same size and then apply technique to lessen the quantity of grey levels in each block while it try to maintain the same standard deviation and same mean. Another version of BTC is Absolute Moment Block Truncation Coding. The lesser the block size the higher PSNR we get but in more time.

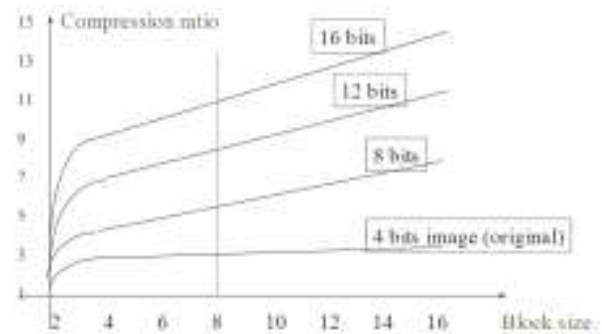

Figure 3: Block size Vs Compression Ratio

As an achievement, the rover images of NASA's Mars Pathfinder ware compressed by the BTC algorithm. IT is a faster approach which provide a satisfactory compression ratio, it preserve edges because it preserves first two samples of its block, so it can be effectively use by edge detection algorithms.

\section{Huffman Coding}

Huffman coding is a form of lossless image compression technique which can also use as entropy coding technique for images. This algorithm was developed by David A.
Huffman, use to eliminate redundancies from the data. It uses tree structure to do so. It use variable-length code like algorithm where code are assign on the basis of frequency of the elements, the element with higher frequency gets the smallest code and the element with lowest frequency gets largest code. There are two major parts of a Huffman coding algorithm, one is generating a Huffman tree and other is traversing that tree to assign codes. It can be use for compressing the image directly but however in MATLAB there is no Huffman in code which can be apply on image because they can only be apply on numeric vector, numeric cell array. Huffman coding is not always as efficient as the other lossless compression algorithm.

\section{Methodology}

The basic methodology of proposed paper is to using EZW and BTC algorithm to compress an image and encourage finding more hybrid algorithms which use 2 or more algorithms to get better result in term of PSNR, CR and time consumption in applying that algorithm.

In the simulation of this proposed algorithm in MATLAB we use a color lena image and convert it into $\mathrm{RGB}$ to $\mathrm{YCbCr}$ image.

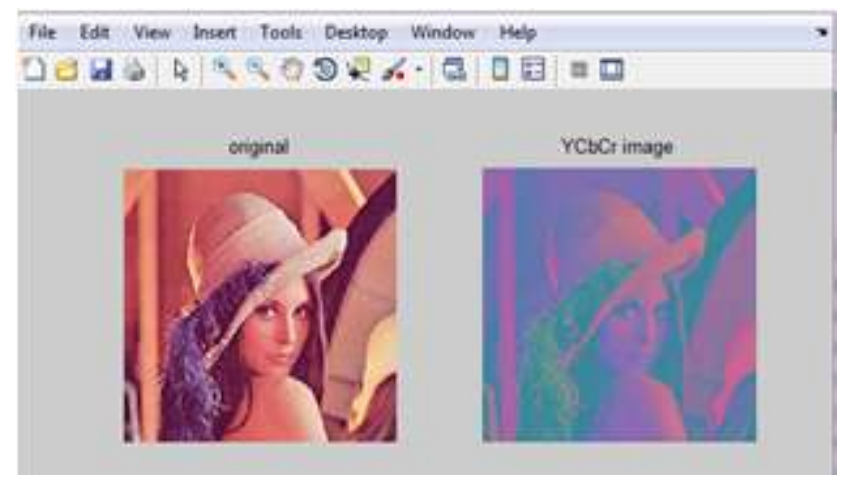

Figure 4: Difference between RGB and YCbCr image

Then we separate the $\mathrm{Y}, \mathrm{Cb}$ and $\mathrm{Cr}$ component of the $\mathrm{YCbCr}$ image and take $\mathrm{Y}$ and apply quantization on it. Quantization is a lossy procedure where we apply some mathematical formula on the image matrix (in MATLAB everything is store in a form of matrix). Then the wavelet decomposition is applied on the quantized matrix. The EZW and then Huffman coding is apply respectively on the result data. And we save the resultant compressed data in a mat file.

Then we take $\mathrm{Cb}$ component and quantize it and apply BTC on the quantized matrix. Then the wavelet decomposition is apply on the quantized matrix. The EZW and then Huffman coding is apply respectively on the result data. And we save the resultant compressed data in a mat file.

Then we take $\mathrm{Cr}$ component and use same procedure we used on $\mathrm{Cr}$ component and store the result. The result of $\mathrm{Y}$, $\mathrm{Cb}$ and $\mathrm{Cr}$ components should store in a same mat file to get a single compressed data.

We do not apply BTC on Y component because it will reduce the PSNR but we apply it on $\mathrm{Cb}$ and $\mathrm{Cr}$ because they are too small and that change in the PSNR can neglect on

\section{Volume 6 Issue 12, December 2017}




\section{International Journal of Science and Research (IJSR) \\ ISSN (Online): 2319-7064 \\ Index Copernicus Value (2016): 79.57 | Impact Factor (2015): 6.391}

them but it can increase the Compression Ratio and as a combined result we get better PSNR (Peak Signal to Noise Ratio).

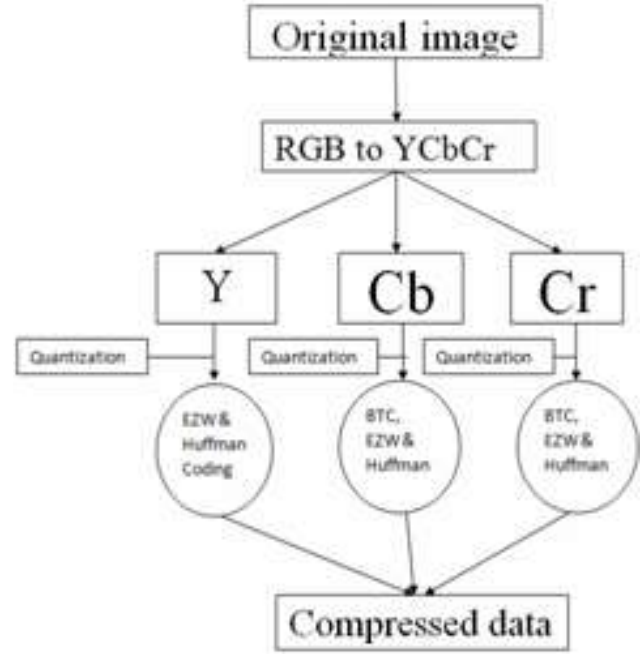

Figure 5: Block diagram of compression procedure in the proposed algorithm

Then we fetch the results from compressed file and separate the result from each component and then we apply decompression on it. First we take result we get from Y component and apply Huffman decoder and then inverse EZW algorithm on it, then we do wavelet reconstruction on it then we dequantize (inverse procedure of quantization) on it and save the decompressed Y component.

Then we take result from $\mathrm{Cb}$ component and apply Huffman decoder and then inverse EZW algorithm on it, and then we do wavelet reconstruction. Inverse BTC apply on that resultant matrix and then we dequantize it and save the $\mathrm{Cb}$ decompressed component. We apply same procedure as $\mathrm{Cb}$ component on $\mathrm{Cr}$ and save the decompressed $\mathrm{Cr}$ component. This data are later on use to reconstruct the $\mathrm{YCbCr}$ image. Then we convert this $\mathrm{YCbCr}$ image into RGB image and get the decompressed original image.

\section{Result}

The result of proposed algorithm or any other algorithm can be deriving in form of two components PSNR and CR.

\section{A. PSNR}

Peak Signal to Noise Ratio shows the quality of decompressed image in form of peak error and can be calculated by following formulas.

$\mathrm{PSNR}=10 \log 10\left(\mathrm{R}^{2} / \mathrm{MSE}\right)$

where

$\mathrm{MSE}=\frac{\sum M, N[l 1(m, n)-l 2(m, n)]^{2}}{M * N}$

\section{B. Compression Ratio}

Compression Ratio is the ratio between original image and compressed image. It tell that the how much our image get compressed or in other words how much percent the compressed data is of original image.

Compression Ratio $=\frac{\text { original image }}{\text { compressed data }}$

For example if the compression ratio is 2 then
$(1 / 2) * 100=50 \%$, means the compressed data is $50 \%$ of the original image.

Percentage of compression $=(1 / \mathrm{CR}) * 100$
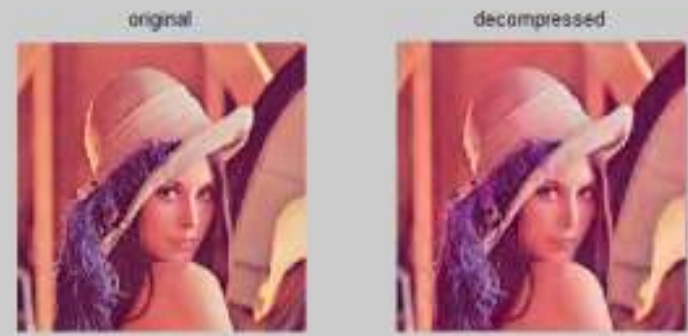

Figure 6: Showing Original image and decompressed image

\section{Future Scope}

The main reason to propose this system is to encourage finding new hybrid system. In future this system can be perform on videos as well (after some changes) and use on live video streaming. And this system can be mixed with other algorithm as well to get better result.

\section{Conclusion}

Images are strong medium when it comes to entertainment, corporate world, research and study but it takes much space to store them on hard disk or on network. The proposed system use a hybrid approach to solve that problem which uses EZW and BTC and Huffman coding to compressed a color image. This system can use on videos and mix with other system to get better results.

\section{References}

[1] LiBin and Meng Qinggang, "An Improved SPIRT Wavelet Transform in the Underwater Acoustic Image Compression", 2nd International Conference on Measurement, Information and Control, IEEE, Harbin, China, page 1315-1318, 2013.

[2] Tobias Lindstrom Jensen, Jan Ostergaard, Joachim Dahl, and Soren Holdt Jensen, "Multiple Description l1-Compression", IEEE Transactions on Signal Processing, VOL. 59, NO 8, August 2011.

[3] Bhawna Rani, R K Bansal and Dr Savina Bansal, "Comparison of JPEG and SPIHT Image Compression Algorithms using Objective Quality Measures", IMPACT, IEEE, page no.90-93, 2009.

[4] F. Khelifi, N. Doghmane and T.Bouden, "Compression Of The Color Images By SPIHT Technique", IEEE, page no.365-366, 2004.

[5] M. Atheeshweri and K. Mahesh, "Video Compression Techniques- A Comprehensive Survey" International journal of advance research in Computer Science and Software Engineering, ISSN 2277-128X, volume 4, issue 1, Jan 2014.

[6] Source, MATLAB R2010a, version 7.10.0.499, 32 bit, Feb. 2010

\section{Volume 6 Issue 12, December 2017}


[7] Ahmed, N. Natarajan, T.Rao, “ Discrete Cosine Transform, ” IEEE Trans. On Computers, vol. C-32, pp. 90-93, Jan. 1974.

[8] Huang Ke-kun, "Improved Set Partitioning in Hierarchical Trees Algorithm Based on Adaptive Coding Order", Journal of ComputerApplication-china, 2012, 32(3):732-735, March 2012.

[9] D.Khosla and A.Kaur, "Design of Hybrid Compression Model using DWT-DCT-Huffman Algorithms for Compression of Bit Stream", International Journal of Engineering Research \&Technology (IJERT) Vol. 1 Issue 5, July - 2012

[10] Antonini, M. Barlaud, M. Mathieu, P. Daubechies,I. “ Image Coding Using Wavelet Transform," IEEE Trans. on Image Processing, Vol. 1, No. 2, pp.205-220.1992

[11] Meng Wang and Qi Han, "An Improved Algorithm of SPIHT based on Human Visual Characteristics", International Journal of Biomedical Science Vol.1, Pg. No.423-335, 2005.

[12] Sonja Grgic, Mislav Grgic, Yuen Cheng "Performance Analysis of Image Compression using Wavelets", IEEE transaction on Industrial Electronics, Vol. 48, No. 3 , Pg. No. 682-695, June 2001.

[13] Sonja Grgic, Marta Mrak, Mislav Grgic, "Comparison of JPEG image coder", Proceedings of the 3rd International Symposium on Video Processing Pg. No. 217-223, 2005.

[14] Jerome M Shapiro "Embedded Image coding using zerotrees of wavelets coefficients", IEEE transactions on signal processing, Vol 41, no. 1 2, Pp.3445-3462, Dec 1993.1

[15] Tarun kumar \& Deepak Chaudhary, "Comparison Study Between ezw, spiht, stw, wdr, 'aswdr' and spiht_3d', International Journal of Scientific \& Engineering Research, Vol 4, pp 710-715, Jan 2009.

[16] H. Kim, C. Choe, and J. Lee, "Fast implementation of 3-D SPIHT using tree information matrix, in Proc. IEEE Int. Geoscience and Remote Sensing Symp., Jul. 2003, vol. 6, pp. 3586-3588.

[17] KP. Soman \& K.I. Ramachandran,"Insight into Wavelets from theory to practice", Prentice Hall India, New Delhi 2002.

[18] Strang, G. and Nguyen, "T. Wavelets and Filter Banks", Wellesley-Cambridge Press, Wellesley, MA, 1996.

[19] N. J. Fliege, "Multirate Digital Signal Processing: Multirate Systems Filter Banks-Wavelets", Wiley \& sons, Jan., 2000.

[20] Ritu Chourasiya and Prof. Ajit Shrivastava, "study of image compression based Transmission algorithm using Spiht for Low bit rate application", An International Journal Advanced (ACIJ), Vol.3, No.6, November 2012. 